\title{
Principle and Experiment of Protein Detection Based on Optical Fiber Sensing
}

\author{
Qi JIANG ${ }^{1 *}$, Mengwei XUE ${ }^{1}$, Pei LIANG ${ }^{1}$, Chengjia ZHANG ${ }^{2}$, \\ Jianqiang LIN $^{2}$, and Jun OUYANG ${ }^{3}$ \\ ${ }^{1}$ School of Control Science and Engineering, Shandong University, Jinan, 250061, China \\ ${ }^{2}$ State Key Lab of Microbial Technology, School of Life Science, Shandong University, Jinan, 250100, China \\ ${ }^{3}$ School of Materials Science and Engineering, Shandong University, Jinan, 250061, China \\ *Corresponding author: Qi JIANGＥ-mail: xuemengweistu@163.com
}

\begin{abstract}
A method for detecting protein molecules based on the tilted fiber Bragg grating (TFBG) surface plasma resonance (SPR) is proposed to achieve the quick online real-time detection of trace amount of proteins. The detection principles of the TFBG-SPR protein molecular probe are analyzed, and its feasibility is demonstrated. The intermediary material between the protein molecules and the golden layer outside of the fiber gratings is cysteamine hydrochloride. When the concentration of the cysteamine hydrochloride solution is $2 \mathrm{M}$, the shift of the TFBG resonance peak is $2.23 \mathrm{~nm}$, illustrating that the cysteamine hydrochloride modifies the gold film successfully. IgG antigen solution is poured on the surface of the cysteamine hydrochloride modifying the gold-deposited TFBG. Finally, antigen-antibody hybridization experiment is carried out with a $10 \mathrm{mg} / \mathrm{mL}$ antibody solution, and after two hours of hybridization the resonance peak of the TFBG shifts $5.1 \mathrm{~nm}$, which validates the feasibility and effectiveness of the TFBG-SPR protein molecular probe.
\end{abstract}

Keywords: Surface plasma resonance; TFBG; protein; molecular probe

Citation: Qi JIANG, Mengwei XUE, Pei LIANG, Chengjia ZHANG, Jianqiang LIN, and Jun OUYANG, "Principle and Experiment of Protein Detection Based on Optical Fiber Sensing," Photonic Sensors, 2017, 7(4): 317-324.

\section{Introduction}

The biosensors based on the surface plasmon resonance (SPR) technology possess prominent advantages over the traditional sensors [1]. The SPR technology, as a new molecular detecting technology, had got a rapid development in recent years. The number of documents in the relevant field has a rapid increase with the vigorous development of the SPR sensor based on the optical fiber and the advent of related products [2]. These years, the SPR sensors have been paid more attention in the field of molecular detecting [3, 4]. Bartley et al. utilized the
SPR technology to design a sensor chip which can quickly identify and screen for unknown receptors and ligands. Yawen Zhang et al. [5] fixed sulfydryl on the golden layer by the self-assembly membrane method, then modified the glucose oxidase and made it a glucose biosensor by fixing chitosan on the surface finally. Tilted fiber Bragg grating (TFBG)-SPR protein molecular probe owns the advantages of label-free detection, real-time monitoring over general molecular probe, and it has been applied in specified recognition and rapidly continuous detection in fields of food safety and drug testing [6, 7]. Recently, Voisin and Pilate et al.

Received: 18 September 2016 / Revised: 12 June 2017

(C) The Author(s) 2017. This article is published with open access at Springerlink.com

DOI: $10.1007 / \mathrm{s} 13320-017-0383-7$

Article type: Regular 
[8] introduced the TFBG with the nanometer-scale golden layer to fabricate a biosensor and detect the alteration of the marker-free human transferrin in the serum, showing that TFBG-SPR sensors had high sensitivity, reversibility, and robustness. Candiani et al. [9] put forward a kind of label-free DNA biosensor based on the double TFBG, and they assembled sensors into peptide nucleic acid probe with a higher sensitivity. TFBG-SPR sensors are suitable for detecting protein interactions. According to the variation in the TFBG cladding mode and SPR theory, the mass and concentration of proteins involved in the reaction can be calculated, and the changes of proteins in the reaction can be detected. This paper puts forward a TFBG-SPR protein molecular detection method and brings an experimental demonstration.

\section{Sensing principles}

Surface plasmon resonance wave is a kind of physical and optical phenomena, which occurs at the interface between the metal and dielectric. The SPR sensor with the effect of the surface plasmon resonance takes excellent advantages on the environment medium sensitive characteristic of refractive index change. It can detect the change of the material on the surface of the metal, and the studies have shown that the SPR effect is very sensitive to the environment temperature change so that the SPR bio-sensor in the study of detection needs a stable environment temperature. The total reflection will occur on the border of thin light medium and dense light medium when the incidence angle of the light is greater than the critical angle and the incident light and reflected light in the interface will interact to produce a standing wave disturbance. When the standing wave extends to light thin medium direction along the boundary face, it will form a special kind of tend towards rapid attenuation of electromagnetic wave, which is called the evanescent wave [10]. When the wave vector of the evanescent wave matches the one of metal film surface electron plasma wave, the energy will be coupled into the surface plasma wave stimulating the SPR effect [11, 12].

With the aid of the deposited biological molecules outside the active golden layer, TFBG-SPR sensors are capable of detecting biological molecules with the nature of the specific identification. In general, bioactive molecules are immobilized on the surface of the golden layer in virtue of sulfydryl (-SH) of the intermediary material substantially. Because the strength of S-H bond is lower than the one of Au-S bond, $\mathrm{S}$ in the solution can be combined with $\mathrm{Au}$, and the biological molecules are fixed on the surface of the golden layer with the formation of the Au-S bonds. Due to the fact that protein molecules are larger, having a complex structure and do not necessarily have sulfydryl, the intermediary material is used to immobilize proteins by linking the golden layer and proteins. In addition to containing sulfydryl, the intermediary material can also form some kind of radical group for bonding the protein molecules. Figure 1 presents the schematic diagram of TFBG-SPR protein molecular probe detection.

Figure 2 reveals the protein detection flow chart of the TFBG-SPR protein molecular probe. It takes a sufficient period of time for immersing the gold-deposited TFBG in the cysteamine hydrochloride solution with a certain concentration. With the generation of gold-sulfur bond, cysteamine hydrochloride is adhered on the surface of the golden layer, and the $\mathrm{NH}_{3} \mathrm{Cl}$ of the cysteamine hydrochloride is ionized into chloride ions and amino. When there is a large number of amino in the solution, it forms amino groups which are capable of adsorbing macromolecular proteins. The attachment of IgG antigen on the surface of the golden layer is based on this property of amino groups. When the corresponding IgG antibodies have combined with $\mathrm{IgG}$ antigen fixed on the golden layer, the properties of the nanometer-scale golden layer will be affected, and so does the nature of the evanescent layer. 
According to the principle of SPR, the wavelength of surface plasmon resonance will be altered when the property of the evanescent layer changes, and the cladding mode of the TFBG transmission spectrum will also shift.

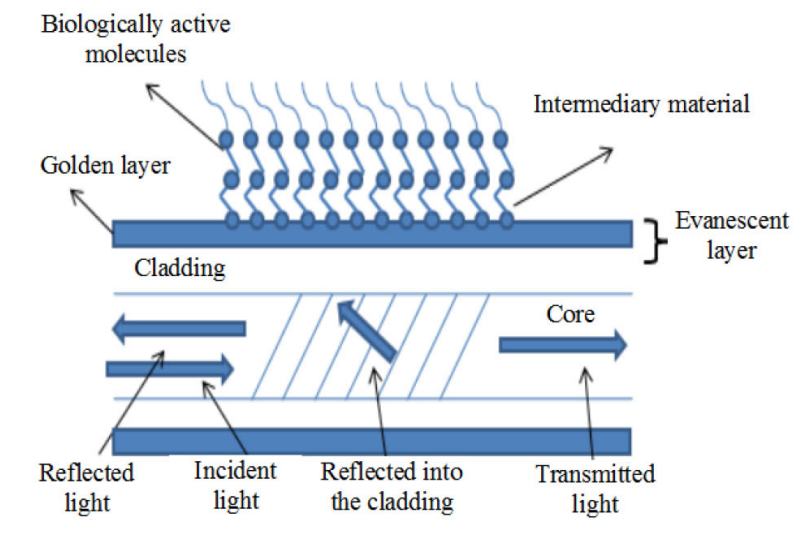

Fig. 1 Schematic diagram of TFBG-SPR protein molecular probe detection.
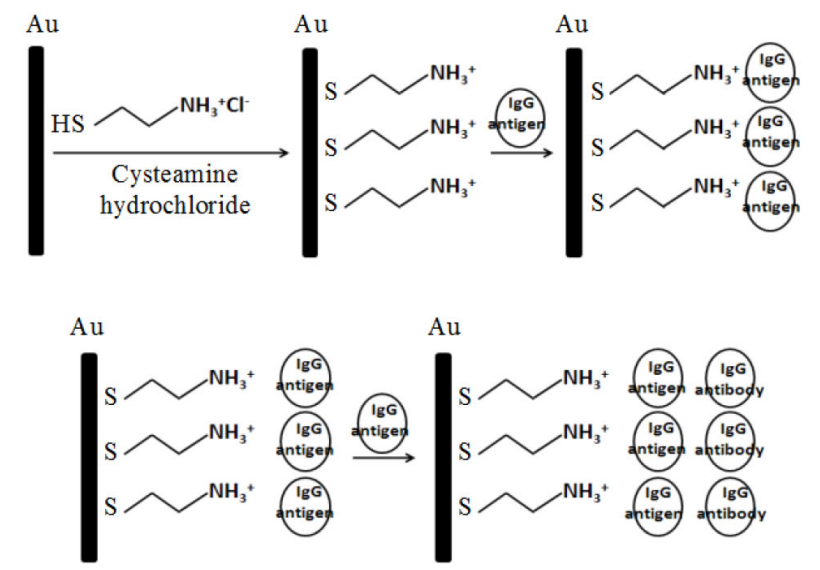

Fig. 2 Protein detection flow chart of the TFBG -SPR protein molecular probe.

\section{Materials and methods}

The probe experiment consisted of cysteamine hydrochloride deposition, fixation of $\operatorname{IgG}$ antigen as well as antigen-antibody hybridization test. Table 1 lists the equipment utilized in the experiment. The experimental materials and reagents are enumerated in Table 2.

\subsection{Cysteamine hydrochloride deposition}

After being rinsed with ultrapure water, TFBG was sonicated with absolute ethanol and then with ultrapure water for $5 \mathrm{~min}$, respectively. Then we began to conduct the cysteamine hydrochloride deposition experiment on the cleaned TFBG. The cysteamine hydrochloride solutions utilized in the experiment had three different concentrations of $20 \mathrm{mmol}, 1 \mathrm{~mol}$, and $2 \mathrm{~mol}$. The deposition experiment lasted more than $12 \mathrm{~h}$ [5] to ensure the cysteamine hydrochloride being fixed on the golden layer adequately. The cleaned TFBG fixed on a glass dish was immersed in the cysteamine hydrochloride solution for $15 \mathrm{~h}$ at room temperature by using a thermostat water bath.

Table 1 Equipmental list.

\begin{tabular}{|c|c|c|}
\hline Instrument & Instrument model & Production unit \\
\hline $\begin{array}{c}\text { ASE broadband light } \\
\text { source }\end{array}$ & ASE-C-M-G-13 & $\begin{array}{c}\text { Shenzhen kuan ti } \\
\text { co., LTD }\end{array}$ \\
\hline Spectrograph & AQ6317B & Yokogawa \\
\hline Electronic balance & YP402 & $\begin{array}{l}\text { Shanghai Jing Hai } \\
\text { Instrument }\end{array}$ \\
\hline Tilted gratings & - & - \\
\hline Abbey refractometer & WAY-2W & Shanghai \\
\hline $\begin{array}{l}\text { Constant temperature } \\
\text { magnetic stirrer }\end{array}$ & & 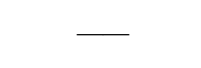 \\
\hline Various glassware & 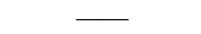 & - \\
\hline
\end{tabular}

Table 2 Materials and reagents required in the experiment.

\begin{tabular}{ccc}
\hline Reagents & Size & Factory unit \\
\hline $\begin{array}{c}\text { Cysteamine } \\
\text { hydrochloride } \\
\text { IgG antigen solution }\end{array}$ & $0.07 \mathrm{~mol} / \mathrm{L}$ & Sangon Biotech Co.,Ltd. \\
IgG antibody solution & $0.35 \mathrm{~mol} / \mathrm{L}$ & Sangon Biotech Co.,Ltd. \\
De-ionized water & - & \\
Ethanol & $95 \%$ & \\
\hline
\end{tabular}

\subsection{Fixation of IgG antigen}

As the cysteamine hydrochloride deposition experiment has been done, the TFBG was rinsed with ultrapure water thoroughly and soaked in water for a while to remove the physically adsorbed cysteamine hydrochloride. Then IgG antigen was diluted into four parts of $1 \mathrm{~mL}$ solutions and stored in the refrigerator for depositing sensitive biological membrane. One of the $1 \mathrm{~mL} \mathrm{IgG} \mathrm{antigen} \mathrm{solutions}$ was injected into the glass dish fixing the TFBG that 
cysteamine modified for IgG antigen deposition, and dried at $4{ }^{\circ} \mathrm{C}$. The above steps were repeated until all of the $1 \mathrm{~mL} \operatorname{IgG}$ antigen solutions have finished depositing.

After the deposition of IgG antigen, the TFBG-SPR protein molecular probe was immersed in the ultrapure water for a while to remove the physical adsorption of cysteamine hydrochloride on the fiber grating and the glass dish. The probe was stored in $4^{\circ} \mathrm{C}$ refrigerator compartment.

\subsection{IgG antigen-antibody hybridization}

To guarantee that the prepared protein sensor can be soaked in the IgG antibody aqueous solution, we diluted the stored IgG antibody by ultrapure water to be $1 \mathrm{~mL}$ IgG antibody solution with a concentration of $10 \mathrm{mg} / \mathrm{mL}$. The corresponding $\operatorname{IgG}$ antibody solution was poured into the glass dish fixing the TFBG modified by IgG antigen, and the experimental temperature was kept constant at $37^{\circ} \mathrm{C}$ by using a thermostat water bath. The whole $\operatorname{IgG}$ antigen-antibody hybridization experiment lasted for about $2 \mathrm{~h}$, and the TFBG transmission spectra were collected automatically every $3 \mathrm{~min}$ during the entire experiment. The experimental site is shown in Fig. 3.

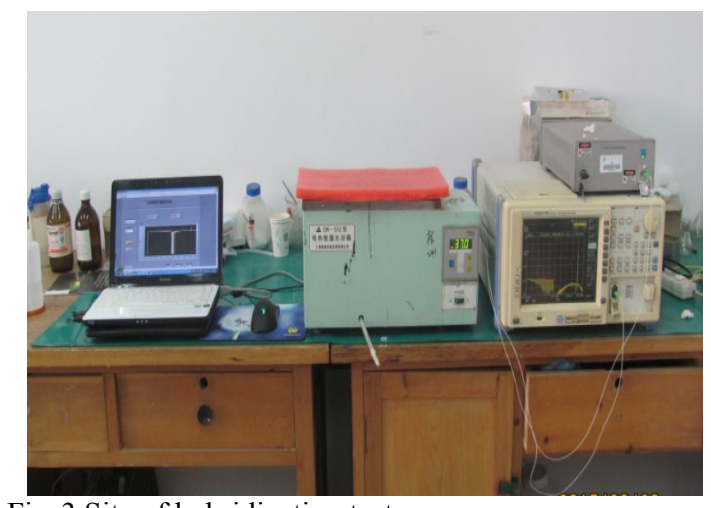

Fig. 3 Site of hybridization test.

\section{Results and discussion}

\subsection{Deposition experiment of cysteamine hydrochloride solution}

\subsubsection{Deposition using $20 \mathrm{mmol}$ cysteamine hydrochloride solution}

At the first period of the experiment, $20 \mathrm{mmol}$ cysteamine hydrochloride solution was utilized, and the gold-coated TFBG was immersed in it for $15 \mathrm{~h}$. The transmission spectra were collected automatically every $3 \mathrm{~min}$, and the data per hour were selected to calibrate by using the MATLAB code.

The cladding mode of the TFBG transmission spectrum almost had no alteration. Taking the surface area of the golden layer into account, the failure of cysteamine hydrochloride deposition was associated with the low concentration of the cysteamine hydrochloride solution. Then new deposition experiment was carried out by using cysteamine hydrochloride solution with a higher concentration.

\subsubsection{Deposition using $1 \mathrm{~mol}$ cysteamine hydrochloride solution}

According to [13], the concentration of the cysteamine hydrochloride solution was increased to $1 \mathrm{~mol}$ for a new deposition experiment. The experiment also lasted for about $15 \mathrm{~h}$, and the final data of the experiment were imported into MATLAB for analysis.

From the results, we concluded that as the experiment went on, the minimum intensity value of the cladding mode of the TFBG transmission spectrum increased gradually, and the cladding mode of the TFBG transmission spectrum shifted to a larger wavelength. Compared with the deposition experiment with $20 \mathrm{mmol}$ cysteamine hydrochloride, there was a certain experimental effect, and the wavelength shift was as small as $0.03 \mathrm{~nm}$.

\subsubsection{Deposition using $2 \mathrm{~mol}$ cysteamine hydrochloride solution}

The same process was repeated using $2 \mathrm{~mol}$ cysteamine hydrochloride solution, and the experimental data were further calibrated by utilizing the same approach. Figure 4 presents the experimental results, and the amplitude change of the most sensitive resonance is indicated by an arrow. 


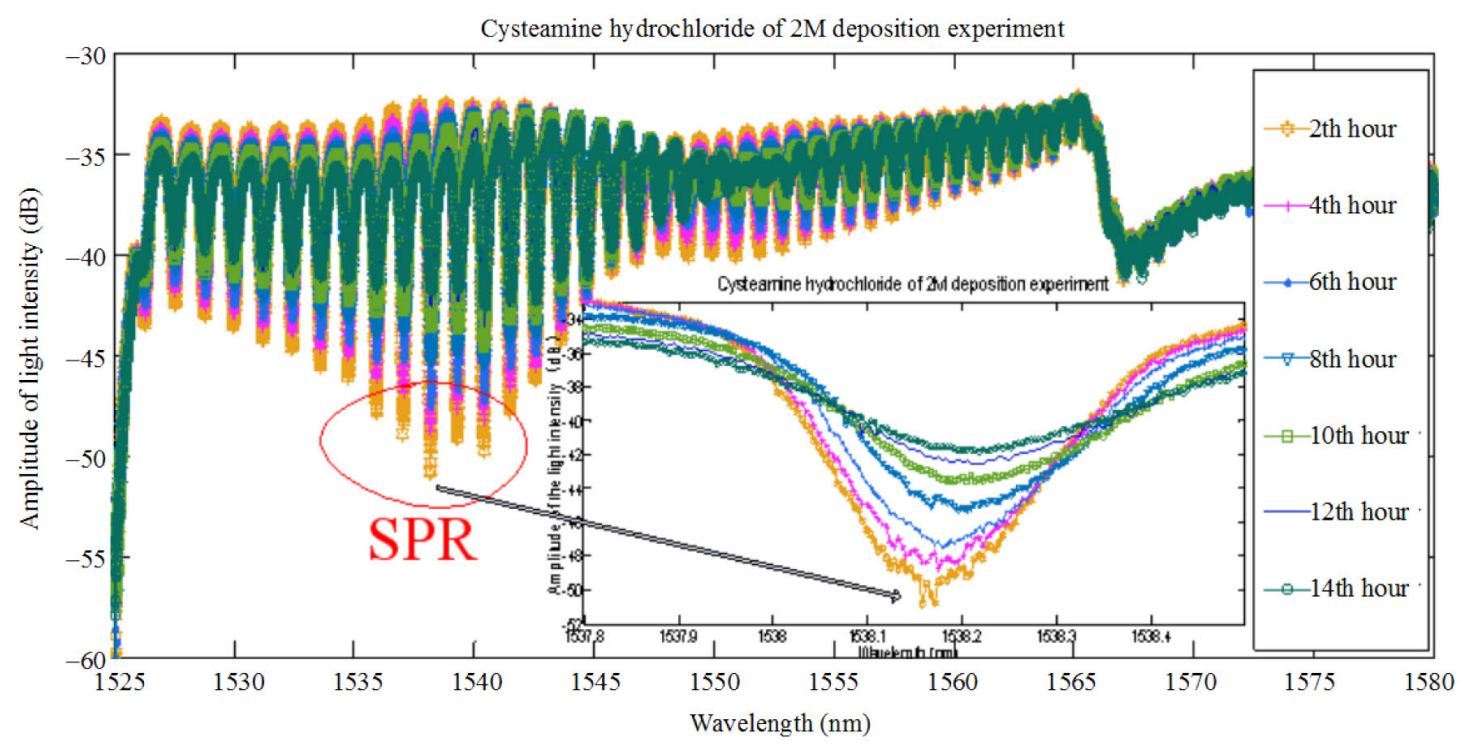

Fig. 4 Results of $2 \mathrm{M}$ cysteamine hydrochloride deposition resonance peak wavelength.

As shown in Fig. 4, the light intensity value of the cladding mode altered obviously. For the purpose of exploring how the transmission spectrum resonance peak changed, the experimental data of the $1 \mathrm{~mol}$ and $2 \mathrm{~mol}$ cysteamine hydrochloride deposition tests in the band between $1526 \mathrm{~nm}$ and $1560 \mathrm{~nm}$ were extracted for configuration. The comparison charts of the variation of the TFBG transmission spectrum resonance peak wavelength are given in Figs. 5 and 6, presenting the amplitudes of the light intensities of TFBG transmission spectrum resonance peaks corresponding to the two cases.

As shown in Figs. 5 and 6, the wavelength of the TFBG transmission spectrum resonance peak increased gradually as the light intensity value became larger. When the concentration of the cysteamine hydrochloride solution was $1 \mathrm{~mol}$, the wavelength changed only $0.03 \mathrm{~nm}$ through the whole experiment, and when the concentration was $2 \mathrm{~mol}$, the wavelength variation was $2.23 \mathrm{~nm}$. The analysis of experimental data demonstrated that cysteamine hydrochloride deposition test had a good result, and a certain number of cysteamine hydrochloride had been fixed on the surface of the golden layer, indicating that the amino groups were capable of adsorbing macromolecular protein.

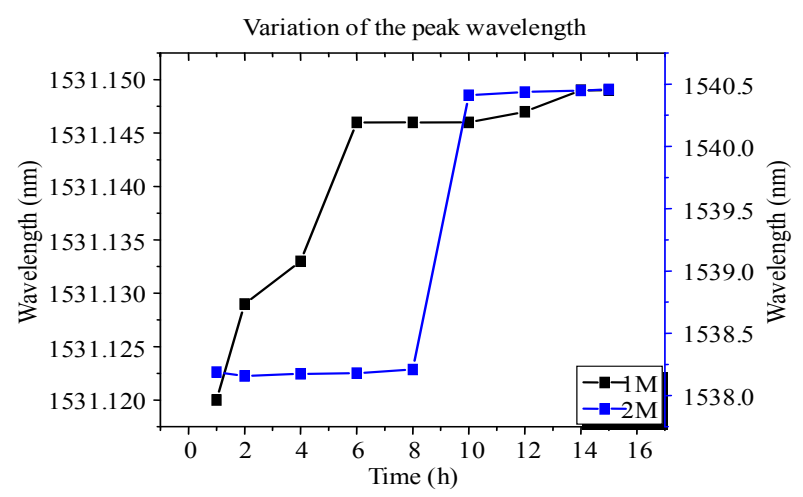

Fig. 5 Variation of the TFBG transmission spectrum.

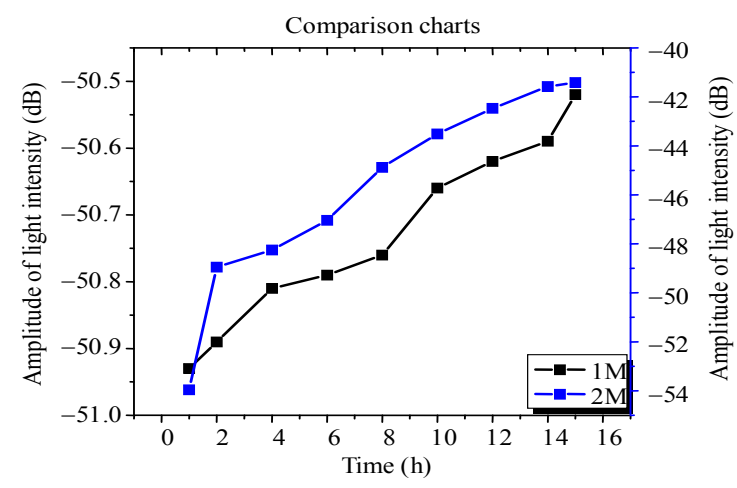

Fig. 6 Amplitude of the light intensity.

\subsection{IgG antigen deposition}

IgG antibody deposition experiment was done as described in Section 3. Figure 7 displays the TFBG 
transmission spectrum after deposition of IgG antigen each time, and the temperature was maintained $4{ }^{\circ} \mathrm{C}$ through the whole process to keep the stability of cysteamine hydrochloride and the activity of IgG antigen. The wavelength of the
TFBG transmission spectrum resonance peak shifted gradually as the IgG antigen deposition was conducting, illustrating that the $\operatorname{IgG}$ antigen was fixed on the golden layer by the dehydration condensation reaction with cysteamine hydrochloride.

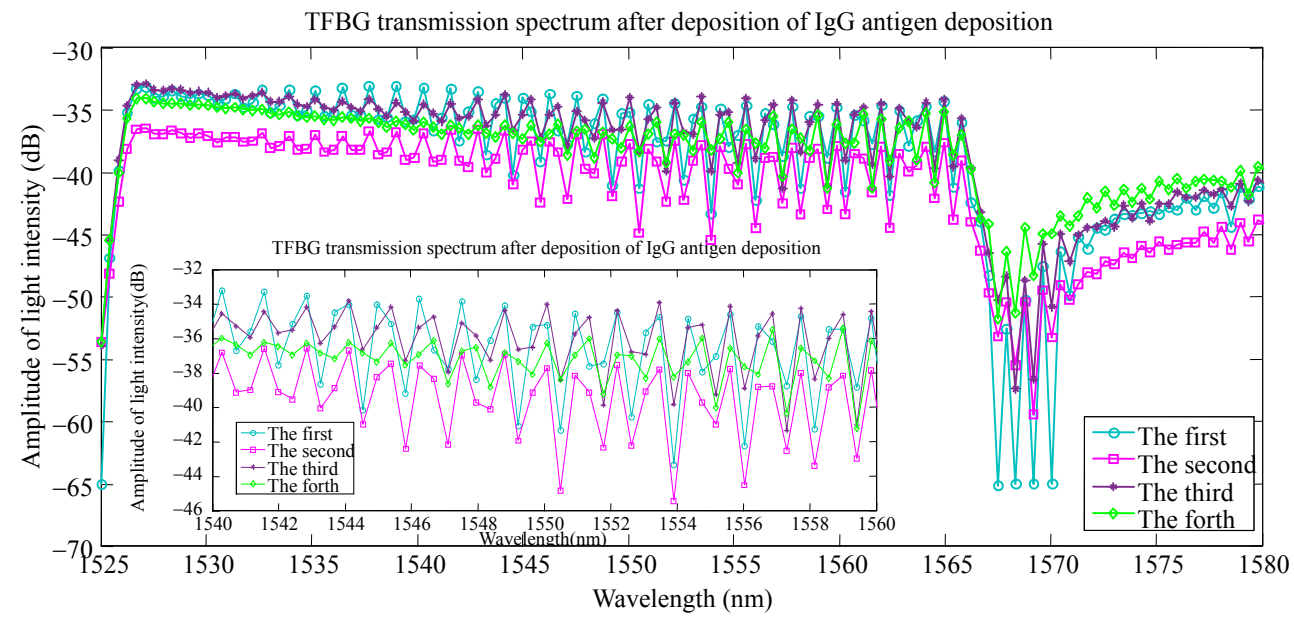

Fig. 7 TFBG transmission spectrum after IgG antigen deposition.

\subsection{IgG antigen-antibody hybridization}

IgG antigen-antibody hybridization experiment was done as described in Section 3. As a kind of special protein, IgG antigen and antibody have a specific recognition features. In general, the temperature range of the antigen-antibody reaction is between $15^{\circ} \mathrm{C}$ and $40^{\circ} \mathrm{C}$ while the optimum temperature is $37^{\circ} \mathrm{C}$. In this experiment, the temperature of the $\operatorname{IgG}$ antigen-antibody hybridization test was suitable for the common protein reaction to ensure the effect of IgG antigen-antibody hybridization test. In addition, the $\mathrm{pH}$ range of the solution required by the antigen-antibody hybridization reaction is between 6 and 9 , and the result of the antigen-antibody reaction will be affected by the over alkali or acid.

The resonance peak wavelength varied over time in the process of $\mathrm{IgG}$ antigen-antibody hybridization experiment, which is presented in Fig. 8. As can be seen from the diagram, the rate of antigen-antibody hybridization was relatively slow at the first half an hour and then higher. After about two hours reaction, the resonance peak wavelength of the cladding mode remained constant basically, illustrating that the reaction was almost finished. The variation of the resonance peak wavelength was $5.1 \mathrm{~nm}$, and the experimental effect was more obvious. The concentration of $\operatorname{IgG}$ antibody utilized in this experiment was $10 \mathrm{mg} / \mathrm{mL}$.

In this work, the data of cysteamine hydrochloride deposition experiment with different concentrations were taken for analysis, and the results showed that when the concentration of the cysteamine hydrochloride solution reached a certain level, the resonance peak wavelength of the TFBG-SPR bimolecular sensor shifted to the larger ones, and the cysteamine hydrochloride deposition was successful. As the cysteamine hydrochloride has been deposited on the surface of the golden layer, IgG antigen could be fixed, and the experimental results illustrated that the attachment of IgG antigen had a good effect. Finally, the analysis of antigen-antibody hybridization experiment indicated that the variation of the resonance peak wavelength was $5.1 \mathrm{~nm}$ and demonstrated the effectiveness of the TFBG-SPR molecular probe. 


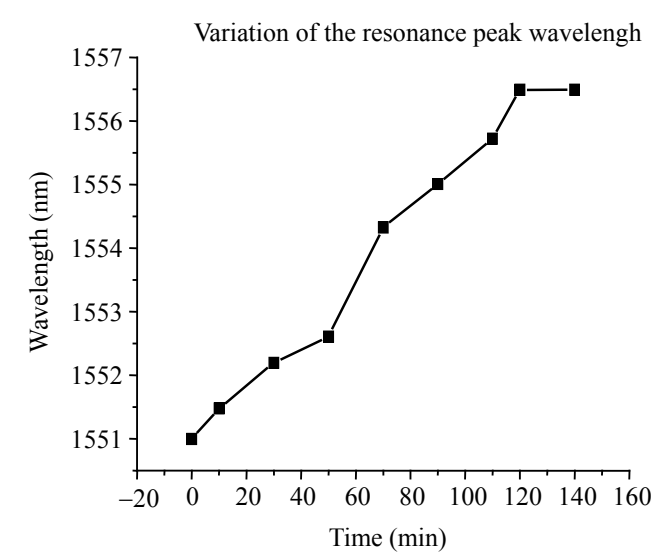

Fig. 8 Resonance peak wavelength changes over time in antigen-antibody hybridization test.

\section{Conclusions}

This paper proposes a method to detect protein molecules based on the TFBG-SPR principles and demonstrates the feasibility by experiments. Firstly, the effectiveness of the TFBG-SPR protein molecular probe was testified through the analysis of detection principles. According to the selected fixing method, cysteamine hydrochloride solutions with different concentrations were used in the cysteamine hydrochloride deposition experiment, and the suitable concentration was selected as $2 \mathrm{M}$. Then IgG antigen could be fixed on the surface of the golden layer by reacting with cysteamine hydrochloride to make it a TFBG-SPR protein molecular probe. Finally, the antigen-antibody hybridization experiment was conducted with the $10 \mathrm{mg} / \mathrm{mL}$ antibody solution, and the experimental data showed that after two hours of hybridization the resonance peak of TFBG shifed $5.1 \mathrm{~nm}$, which validated the feasibility and effectiveness of the TFBG-SPR protein molecular probe. Cysteamine hydrochloride was chosen as the intermediary material to implement the protein molecules fixation on the golden layer of the TFBG. The TFBG-SPR protein molecules probe in this article could basically meet the requirements of low cost, miniaturization, and specificity in the practical application of the biosensor. It also enables the real-time monitoring for the specific recognition of protein molecular, with a higher sensitivity and good prospects for development.

\section{Acknowledgment}

This work was financially supported by the National Nature Science Foundation of China (Nos. 61271073 and 61473175) and was supported by the Fundamental Research Funds of Shandong University (No. 2015JC040).

Open Access This article is distributed under the terms of the Creative Commons Attribution 4.0 International License (http://creativecommons.org/ licenses/by/4.0/), which permits unrestricted use, distribution, and reproduction in any medium, provided you give appropriate credit to the original author(s) and the source, provide a link to the Creative Commons license, and indicate if changes were made.

\section{References}

[1] J. Homola, S. S. Yee, and G. Gauglitz, "Surface plasmon resonance sensors: review," Sensors \& Actuators B - Chemical, 1999, 54(1-2): 3-15.

[2] B. B. Shuai, L. Xia, Y. T. Zhang, C. Zhou, and D. M. Liu, "Principle and study progress of surface plasmon resonance sensors based on gratings," Laser \& Optoelectronics Progress, 2011, 48(10): 100502.

[3] M. J. Xu, "Probe technology and its application in the detection of food pathogens," Food \& Fermentation Industries, 2001, 2: 66-71.

[4] S. B. Xiao, "Detection of transgenic maize using surface plasmon resonance (SPR) DNA biosensor," Journal of Maize Sciences, 2009, 2: 38-43.

[5] Y. W. Zhang, Y. Q. Li, W. J. Wu, Y. R. Jiang, and B. R. $\mathrm{Hu}$, "Chitosan coated on the layers' glucose oxidase immobilized on cysteamine Au electrode for use as glucose biosensor," Biosensors and Bioelectronics, 2014, 60: 271-276.

[6] M. W. Xue, Q. Jiang, C. J. Zhang, and J. Q. Lin, “A kind of biomolecular probe sensor based on TFBG surface plasma resonance," Photonic Sensors, 2015, 5(2): 102-108.

[7] V. Voisin, J. Pilate, P. Damman, P. Megret, and C. Caucheteur, "Macromolecular detection of streptavidin with gold-coated tilted FBG refractometers," SPIE, 2012, 8439: 843904-1843904-7.

[8] V. Voisin, J. Pilate, P. Damman, P. Megret, and C. Caucheteur, "Highly sensitive detection of molecular interactions with plasmonic optical fiber 
grating sensors," Biosensors \& Bioelectronics, 2014, 51(2): 249-254.

[9] A. Candiani, M. Sozzi, A. Cucinotta, S. Selleri, R. Veneziano, R. Corradini, et al., "Optical fiber ring cavity sensor for label-free DNA detection," IEEE Journal of Selected Topics in Quantum Electronics, 2012, 18(3): 1176-1183.

[10] H. J. Huang, J. H. Zhai, B. Q. Ren, R. F. Yang, Y. K. Zhao, Z. G. Cheng, et al., "Fiber-optic evanescent wave biosensor and its application," Acta Optica Sinica, 2003, 23(4): 451-454.

[11] E. Kretschmann and H. Raether, "Radiative decay of non-radiative surface plasmons excited by light," Zeitschrift Für Naturforschung A, 1968, 23(12): 2135-2136.

[12] J. Čtyroký, F. Abdelmalek, W. Ecke, and K. Usbeck, "Modelling of the surface plasmon resonance waveguide sensor with Bragg grating," Optical \& Quantum Electronics, 1999, 31(9-10): 927-941.

[13] W. Y. Hu and L. J. Fu, "Gold surface acridine orange self-assembled membrane preparation and determination of proteins," Journal of Shanxi University: Natural Science Edition, 2010, 33(1): 116-119. 